\title{
Assessment of the Left Ventricle Wall Motion Using Tagged Magnetic Resonance Imaging Data (tMRI)
}

\author{
Mohammed D. Alenezy ${ }^{1,2}$ \\ ${ }^{1}$ Basic Science Department, Faculty of Education, The University of Dammam, Dammam, KSA \\ ${ }^{2}$ Physics Department, Faculty of Science, Aljouf University, Al Jouf, Skaka, KSA \\ Email: mdahawy@gmail.com
}

Received 8 October 2015; accepted 6 November 2015; published 9 November 2015

Copyright (C) 2015 by author and Scientific Research Publishing Inc.

This work is licensed under the Creative Commons Attribution International License (CC BY). http://creativecommons.org/licenses/by/4.0/

(c) () D Den Access

\begin{abstract}
In this paper, the Radial Strain (RS) and Strain Rate (SR) was calculated using tagged MRI (tMRI) data. Using tagged magnetic resonance imaging (tMRI), the left ventricle short axis of five healthy adults (three men and two women) and four healthy male rats was imaged during diastolic and systolic phases on the mid-ventricle level. The RS and radial SR of the left ventricle were calculated at the mid-ventricular level of the cardiac cycle. The peak RS for rat and human heart was found to be $46.8 \pm 0.68$ and $40.7 \pm 1.44$, respectively, and it occurred at $40 \%$ of the cardiac cycle for both human and rat hearts. The peak systolic and diastolic radial SR for human heart was 1.10 $\pm 0.08 \mathrm{~s}^{-1}$ and $-1.78 \pm 0.02 \mathrm{~s}^{-1}$, respectively, while it was $4.25 \pm 0.02 \mathrm{~s}^{-1}$ and $-5.16 \pm 0.23 \mathrm{~s}^{-1}$, respectively for rat heart. The results show that tMRI data can be used to characterize the cardiac function during systolic and diastolic phases of the cardiac cycle, and as a result, it can be used to evaluate the cardiac motion by calculating its RS and radial SR at different locations of the cardiac wall during both diastolic and systolic phases. This study also approves the validity of the tagged MRI data to accurately describe the radial cardiac motion.
\end{abstract}

\section{Keywords}

Left Ventricle, Radial Strain, Radial Strain Rate, Tagged MRI

\section{Introduction}

The typical orientation of the myocardial layers and its changes during systolic and diastolic phases is related to the left ventricle (LV) wall deformation in three directions: radial, circumferential, and longitudinal changes [1] 
[2]. The knowledge of the LV structure is the fundamental to understand these three different motions of the LV wall. In the past few decades, to track the LV motion, markers had to be implanted on the LV wall; and measurements could only be done in animals or patients during cardiac surgery. This invasive method usually affects the accuracy of LV motion. Various measurement methods were used to evaluate the LV function; Noninvasive imaging techniques became available to characterize LV wall motion. Among these techniques, cardiac magnetic resonance is the gold method, over the past years, efforts were focused to develop a standardized method to assess the LV motion and have facilitated the interpretation of LV motion before it could be used for cardiac evaluation [3] [4].

Cardiac Magnetic Resonance (CMR) with tissue tagging [5] is used as the unique approach, because it is able to create noninvasive marks over the entire myocardium tissue in any anatomical plane, it can be tracked throughout the cardiac cycle. The widespread availability of CMR with tissue tagging tool may lead to a fast introduction of LV motion as a clinical measure for detection of myocardial abnormality. However, before LV motion can be used as a clinical tool, the physiology of the LV motion should be well understood first. The structural arrangement of the cardiac fibers is believed to be both energetically optimal and important for equal redistribution of LV stresses and strains when isovolumic contraction (IVC) begins.

The LV wall motion of a healthy heart in short-axis view is very complicated due to the radial and rotational components during both systolic and diastolic motion of the cardiac cycle [2]. However, the LV wall motion at mid-ventricle is mostly in radial direction, which means there is an isotropic contraction with a very small amount of rotational motion. Because most of the stroke volume is produced by LV wall thickening in the radial direction, longitudinal subtle regional dysfunction has relatively little impact on LV global function [6].

This study was under taken to calculate the Radial Strain (RS) and radial Strain Rate (SR) of human and rat subjects using tagged magnetic resonance imaging (tMRI) acquired from healthy human and healthy rat heart modality. Considering that the rotation of the LV wall is negligible at the mid-ventricle level, the present study deals only with images of the mid-ventricle level to calculate RS and radial SR of LV.

Several conditions for studying this radial motion should be taken into account and it can be summarized as [7]:

1) LV shape is a cylindrically symmetric along its long-axis;

2) Origin of the coordinates is located at the center of the LV wall in the short axis view;

3) The systolic contraction corresponding to the end-diastolic phase occurs at $t=0$, where, the LV wall appears at its maximum size and experiences no deformation or rotation motion;

4) The shortening of the LV wall along its long axis only produces negligible amount of out-of-plane motion.

\section{Methods}

Strain: Strain can be defined as the deformation of an object, normalized to its original shape. In a one-dimensional, the only deformation of the object is lengthening or shortening. This is illustrated in Figure 1(a). The relative amount of deformation is defined as strain. Strain, for which the symbol $\varepsilon$ is used, can be written [8]

$$
\varepsilon=\frac{L-L_{0}}{L_{0}}
$$

With $L$ is the length of the object after deformation and $L_{0}$ its original length. By convention, Equation (1) is defined in such a way that lengthening is represented as a positive value for strain, while shortening is represented by a negative value. When the length of the object is not only known before and after deformation but also during the deformation process, the instantaneous strain can be defined Where $L(t)$ is the length of the object at time instance $t$ and $L\left(t_{0}\right)$ is initial length. In other words, the instantaneous deformation is expressed relative to the initial length; this is the Lagrangian strain. The deformation can also be expressed relative to the length at a previous time instance:

$$
\varepsilon(t)=\frac{L(t)-L\left(t_{0}\right)}{L\left(t_{0}\right)} .
$$

SR: SR is the speed at which deformation (i.e., strain) occurs.

The relationship between strain and wall thickening: for the heart, wall thickening $(W T)$ is defined as: 


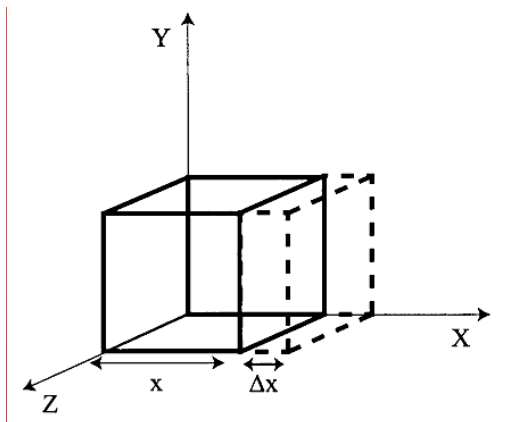

(a)

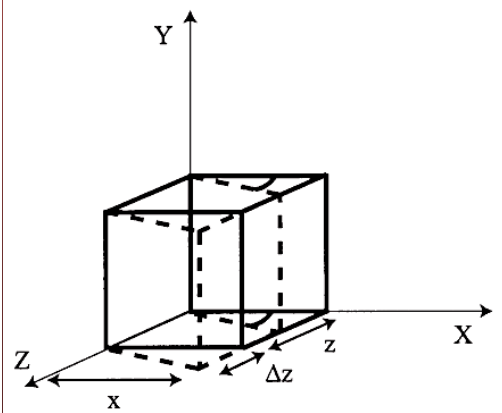

(c)

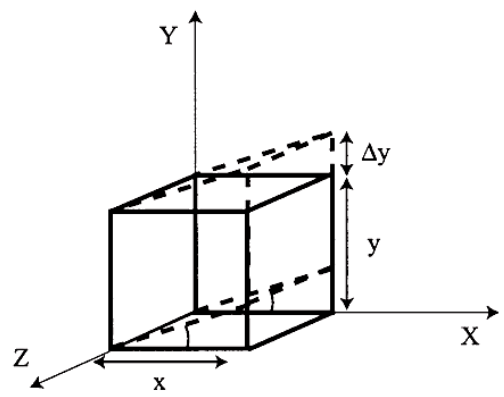

(b)

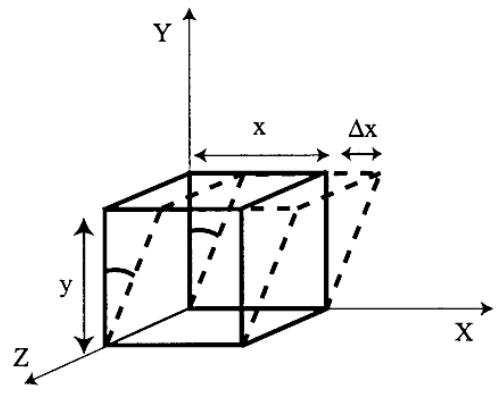

(d)

Figure 1. Deformation of a 3D object is described by three normal and six shear strain components. One normal component (a) and three shear components [_yx (b), _zx (c) and $\left.\_x y(d)\right]$ are illustrated here.

$$
W T=\frac{T_{E S}-T_{E D}}{T_{E D}}
$$

where $T_{E S}, T_{E D}$, and $W T$ are, end-systolic, end-diastolic, and wall thickness, respectively. Comparing this expression with to Equation (1), wall thickening is the cardiac strain measured in one dimension. This is nothing but Lagrangian strain with the (fixed) end-diastolic wall thickness. The rate at which the myocardial wall contract and expand is the one dimension SR [8].

The Heart coordinate system: Figure 2(a), shows the local heart coordinate system which be defined as follows: For each point to be interrogated in any myocardial wall, three mutually perpendicular axes can be defined: The radial (R), The longitudinal (Lo) and The circumferential (C) axis. This local coordinate system is illustrated in Figure 2(b). Cardiac tagged MRI (tMRI) modality was used for visualizing the regional dynamics of the beating hearts in healthy humans ( $\mathrm{n}=5 ; 3$ males and 2 females) and four healthy male rats. The procedures for the scans performed in this work were approved by the institutional committee.

The tagged image contains a mesh of dark lines for labeling the material coordinates of the underlying myocardial tissue. Figure 3 shows the selected area of interest for human heart as a short axis view of the left ventricle while undergoing contraction. As seen in the images, the tag lines warp with the motion of the LV wall and so provide critical information about the regional contraction and viability of the myocardial tissue.

Human tMRI protocol [7]: tMRI data were acquired using a 3 T clinical scanner (Model Signa HDxt, GE Healthcare, Inc., Milwaukee, Wisconsin) with HD cardiac coil. The acquisition was gated using ECG and respiratory signals and the imaging parameters were as follows: $\mathrm{TR} / \mathrm{TE}=4.8 / 2.2 \mathrm{~ms}$, flip angle $=10 \mathrm{~ms}$, bandwidth $=$ $498 \mathrm{~Hz}$, number of averages $=1$, field of view $=350 \times 400 \mathrm{~mm}^{2}$ and image matrix $=372 \times 512$ pixels yielding an in-plane resolution of $0.941 \times 0.781 \mathrm{~mm}^{2}$. The separation between tag lines was $7.0 \mathrm{~mm}$ and the tag width was $1.5 \mathrm{~mm}$. The LV was divided into 12 slices along its long axis, as shown in Figure 4(a). Each slice thickness was $8 \mathrm{~mm}$ without gaps in between. The slices fully covered the apex, mid-ventricle and base levels and the 

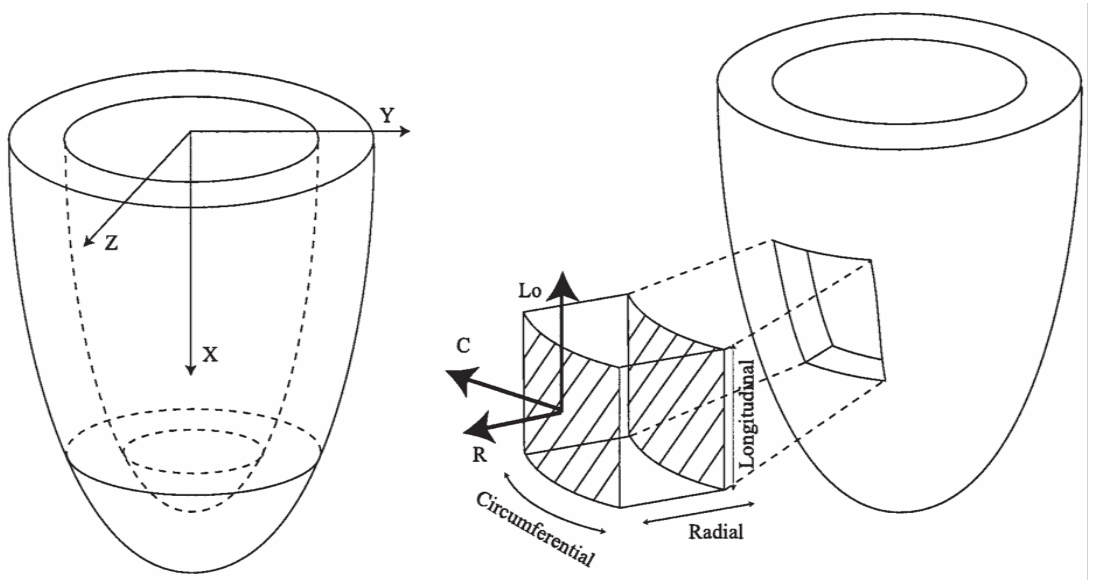

Figure 2. Illustration of LV of the heart showing the three coordinate axis: The radial (R), the longitudinal (Lo) and the circumferential (C) axis.

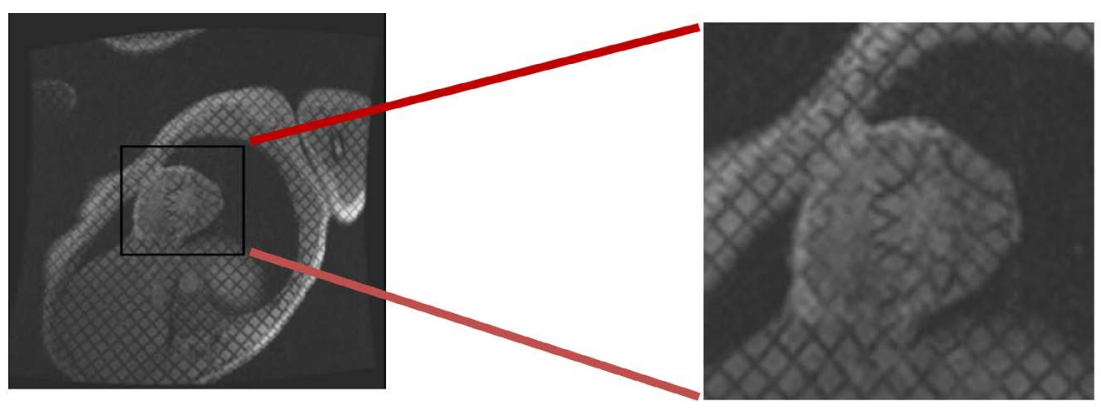

Figure 3. This figure shows the selected area of interest for human heart as a short axis view of the left ventricle while undergoing contraction (300\%).

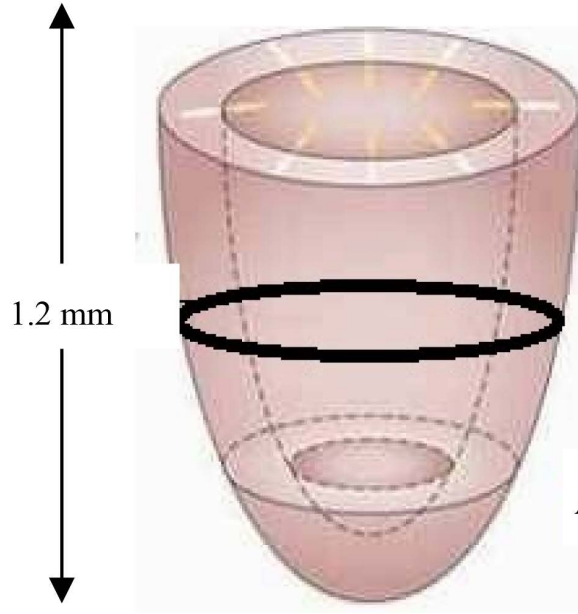

(a)

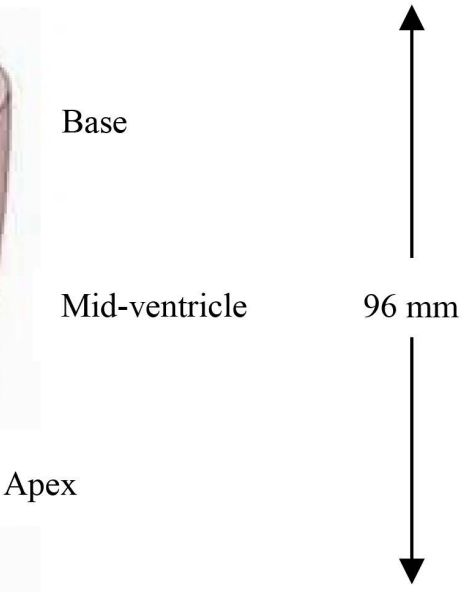

(b)

Figure 4. The slice division of long axis view for: (a) rat LV and (b) human LV.

LV were viewed from the head-to-toe direction. Cardiac cycle period was divided into 11 equal-spaced time intervals and the first frame corresponded to the end-diastole when the LV was fully diluted.

Animal tMRI protocol: Regarding animal protocol, cardiac tMRI data were acquired from four male Sprague-Dawley rats, with the procedures described in [9]. The rats were anesthetized using $1.5 \%$ isoflurane in a mixture of $60 \%$ air and $40 \%$ oxygen. A 9.4 T horizontal bore scanner with a $60 \mathrm{~mm}$ radio frequency volume coil 
was used for MRI. ECG gated gradient echo based. Tagged images were collected for short-axis planes of the heart. The imaging parameters were: TR/TE $=25.00 / 2.44 \mathrm{~ms}$, the number of averages $=1$, the field of view $=60 \times$ $60 \mathrm{~mm}^{2}$, and the image matrix $=256 \times 256$ pixels yielding an in-plane resolution of $234 \times 234 \mu \mathrm{m}$. Separation between tag lines was $0.8 \mathrm{~mm}$ and the width of each tag line was $0.3 \mathrm{~mm}$. The rat LVs was divided into 7 slices along the long axis as shown in Figure 4(a). The thickness of the slice is $2.0 \mathrm{~mm}$ with no space between the slices. All rats were imaged at the mid-ventricular level. To study the LV wall motion in a full cardiac cycle, the whole cardiac cycle was divided into 10 equal-spaced phases and one tMRI image of the heart was acquired at each phase. The first phase was chosen near end diastole phase when the LV was fully diluted while phase 5 was chosen at end systole when the LV experienced the maximal deformation. All the experimental procedures were approved by the Institutional Animal Care and Use Committee at the University of Kansas Medical Center (UMKC).

Preprocessing of the tMRI data [7]: first the center of the LV wall was located then the epicardial and endocardial radii in every $1^{\text {st }}$ end-diastolic image from different slices was calculated. To do that, at the slice corresponding to the mid-ventricle level, as an example, a series of stroboscopic images were first chosen from the underlying slice location. The $1^{\text {st }}$ image frame in the series was displayed to depict the end-diastolic LV on the computer screen. The LV wall on this image showed the largest cross sectional dimension compared to other images in the series. Then, a circle was aligned with the epicardium of the LV wall. The center and radius of the circle were taken as the center and epicardial wall radius $\left(\mathrm{r}_{\mathrm{epi}}\right)$ of the $\mathrm{LV}$ at the end-diastole. $\mathrm{r}_{\text {endo }}$ was similarly estimated from a smaller circle by aligning its circumference with endocardium (Figure 5).

Image analyses: Images were analyzed using the Image $\mathrm{J}$ software [10] at 300\% precision zoom. The cardiac cycle was divided into ten phases. The blood filled LV appeared hyper-intense on images, thus providing excellent contrast for manually tracing the boundary of LV endocardium and epicardium. For each LV slice, the slice two areas is calculated by manually tracing the blood disc (inner area) and the outer area (as shown in Figure 5) using the pixel to area conversion factor of 1 pixel $/ 0.23 \mathrm{~mm}^{2}$ for rat heart and 1 pixel $/ 0.74 \mathrm{~mm}^{2}$ for human heart. The endocardium and epicardium radius was then calculated for each phase. The Radial Strain was then calculated by taking the difference between the current phase radius and the minimum phase radius and divide it by the minimum phase radius (as in Equation (2)). These calculations were repeated for all ten phases of the cardiac cycle. The phases corresponding to the largest and smallest LV endocardium radius were chosen to be representative of end-systole and end-diastole, respectively.

\section{Results}

In the tagged MRI human images, the $6^{\text {th }}$ slice position corresponded to the mid-ventricle level, while it was the $4^{\text {th }}$ slice for tagged MRI animal images (Figure 4). An example of the short axis images of the human left ventricle and the rat left ventricle at end systolic and end diastolic phase at mid ventricular level is shown in Figure 6.

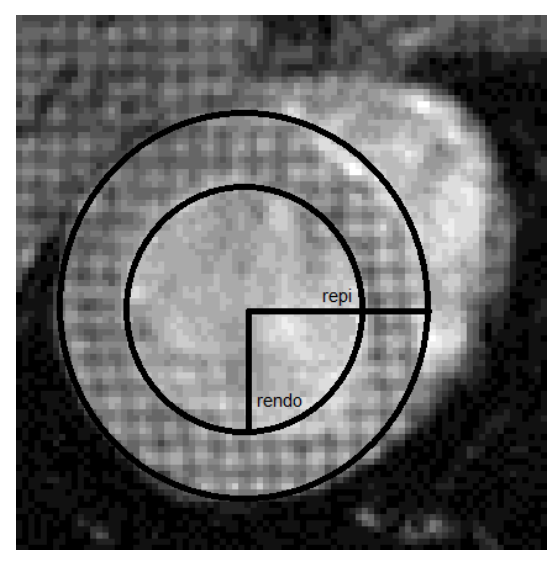

Figure 5. This figure shows the selection of the center of $\mathrm{LV}$ and the how the outer (epi) and inner (endo) radius of the LV of short axis view of the rat during end diastolic phase. 


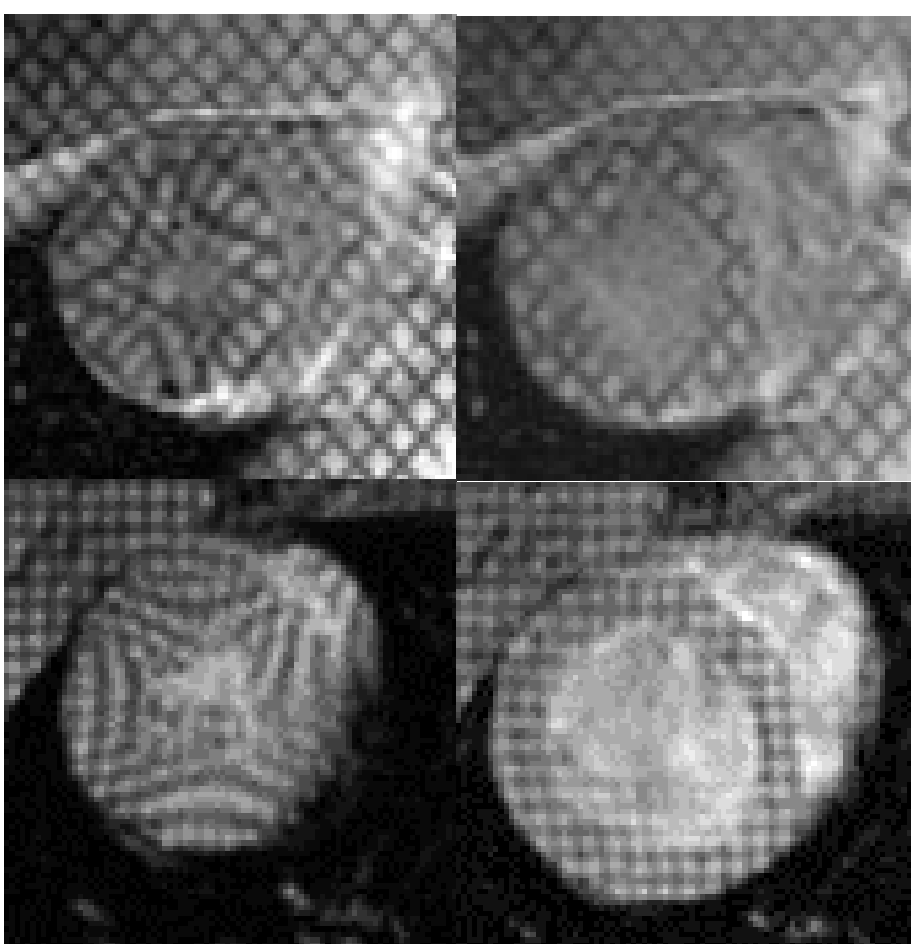

(a)

(b)

Figure 6. Short axis view of LV for human heart (upper) and rat heart (bottom) at (a) end-systole and (b) end-diastole.

Table 1 and Table 2 illustrate the results of Radial Strain and Radial SR for human and Rat heart, respectively. The left ventricle Radial Strain and radial SR were calculated at the mid-ventricular level during the complete cardiac cycle. The maximum Radial Strain for the human and rat LV wall were $40.7 \pm 1.44$, and $46.8 \pm$ 0.68 respectively, and it occurs at $40 \%$ of the cardiac cycle for both human and rat LV wall. The maximum diastolic and systolic radial SR for human LV wall were $-1.78 \mathrm{~s}^{-1} \pm 0.02 \mathrm{~s}^{-1}$ and $1.10 \pm 0.08 \mathrm{~s}^{-1}$ respectively, while for rat $\mathrm{LV}$ wall it was $-5.16 \pm 0.23 \mathrm{~s}^{-1}$ and $4.25 \pm 0.02 \mathrm{~s}^{-1}$ respectively. There is no significant difference between the Radial Strain of human $(40.7 \pm 1.44)$ and rat $\mathrm{LV}$ wall $(46.8 \pm 0.68)$; however, there is a significant difference between radial SR in human and rat LV wall for both systolic $\left(-1.78 \mathrm{~s}^{-1} \pm 0.02 \mathrm{vs}-5.16 \pm 0.23 \mathrm{~s}^{-1}\right)$ and diastolic $\left(1.10 \pm 0.08 \mathrm{~s}^{-1}\right.$ vs $\left.4.25 \pm 0.02 \mathrm{~s}^{-1}\right)$ phases.

Figure 7 shows the change of Radial Strain during the cardiac cycle for human and rat heart. The two figures has almost the same trend, when we consider the Radial Strain change; however, the cardiac cycle period difference between human and rat heart causes the delay in the Radial Strain change during systolic phase for human heart.

In Figure 8, the radial SR change for human and rat heart during the cardiac cycle is shown, the systolic radial SR for rat heart is almost three times that of the human heart, where, the diastolic radial SR for rat is almost four times that of the human heart, this may be due to the difference between the period of the cardiac cycle for rat and human heart.

\section{Discussion}

The similarities of Radial Strain between human and rat left ventricle are in agreement with other noninvasive studies [11]-[14]. Using tMRI as a noninvasive technique to calculate Radial Strain for human and rat heart gives another dimension to the golden role of tMRI in evaluating important function of the cardiac motion such as Radial Strain and Radial SR which can be used as an indicator of the cardiac abnormalities.

The agreement of the Radial Strain values between human and rat heart is another indicator of this accuracy. Because of the heart size and cardiac cycle period difference between human and rat, radial SR show significant difference between both human and rat heart during diastolic and systolic phases, this difference is in good 
Table 1. Data of radial strain and SR for human heart.

\begin{tabular}{ccccc}
\hline SD_SR $^{* *}$ & SR $\left(\mathrm{s}^{-1}\right)$ & SD_RS $^{*}$ & Radial Strain & Time (msec) \\
\hline 0.03 & 0.39 & 0.90 & 19.46 & 83 \\
0.04 & 0.99 & 0.64 & 20.11 & 249 \\
0.08 & 1.10 & 0.43 & 23.36 & 332 \\
0.01 & -0.89 & 0.74 & 31.60 & 415 \\
0.01 & -1.66 & 1.44 & 40.71 & 498 \\
0.02 & -1.78 & 1.37 & 33.34 & 581 \\
0.02 & 0.14 & 1.32 & 19.54 & 664 \\
\hline
\end{tabular}

*SD_RS standard deviation of the Radial Strain, ${ }^{* *}$ SD_SR standard deviation of SR.

Table 2. Data of radial strain and SR for rat heart.

\begin{tabular}{ccccc}
\hline SD_SR $^{* *}$ & SR $\left(\mathrm{s}^{-1}\right)$ & SD_RS $^{*}$ & Radial Strain & Time (msec) \\
\hline 0.13 & 0.07 & 0.13 & 27.4 & 25 \\
0.14 & 4.10 & 0.14 & 27.5 & 50 \\
0.02 & 4.25 & 0.02 & 37.0 & 75 \\
0.17 & -4.36 & 0.17 & 46.8 & 100 \\
0.23 & -5.16 & 0.23 & 36.7 & 125 \\
0.03 & -1.75 & 0.03 & 24.8 & 150 \\
\end{tabular}

*SD_RS standard deviation of the Radial Strain, ${ }^{* *}$ SD_SR standard deviation of SR.
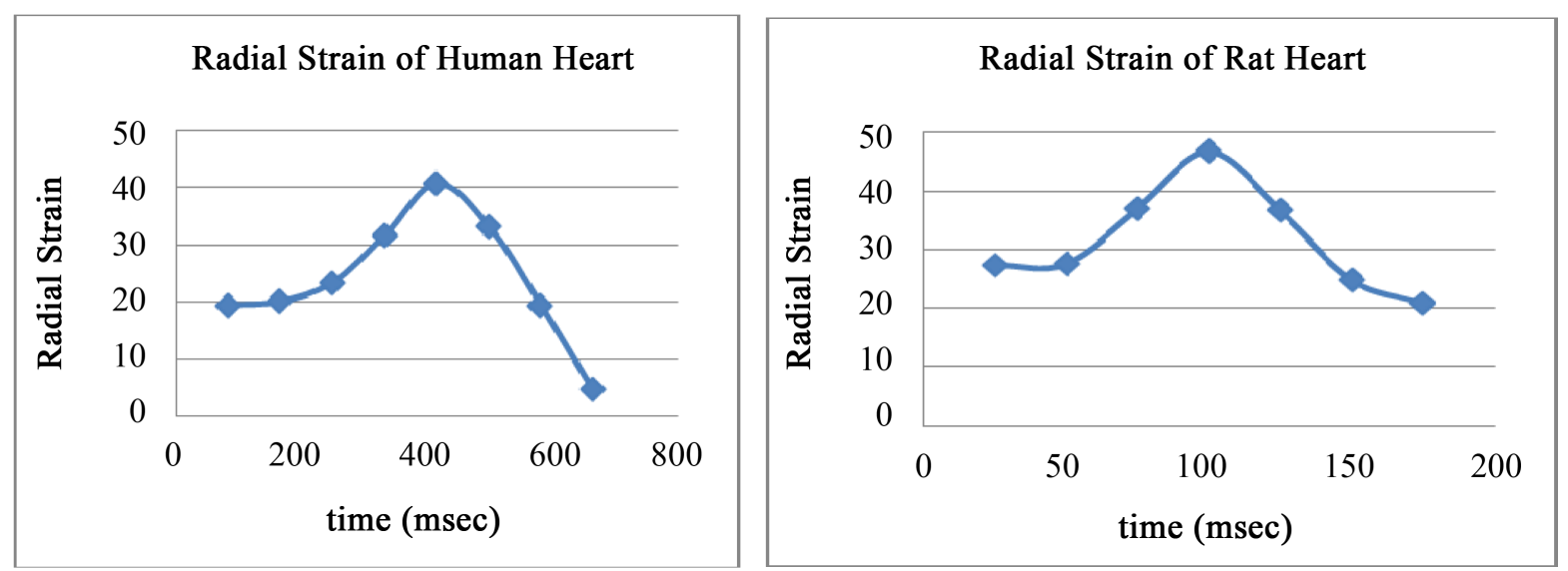

Figure 7. The radial strain change for human and rat heart during the cardiac cycle.

agreement with other noninvasive studies [15] [16]. The difference may be due to the difference in cardiac cycle period between human and rat heart (800 - $1000 \mathrm{~ms}$ vs 225 - $300 \mathrm{~ms}$ ).

The use of the tMRI data was essential in validating the values of the Radial Strain and radial SR of the LV wall during its contraction phase. Radial Strain and Radial SR could be calculated using images acquired with other imaging techniques such as cardiac cine imaging or echography using image intensity features. However, alterations in the intensity pattern of the cine images during the cardiac cycle and speckle decorrelation of the myocardial echogeneity in these methods could result with less precision values. 

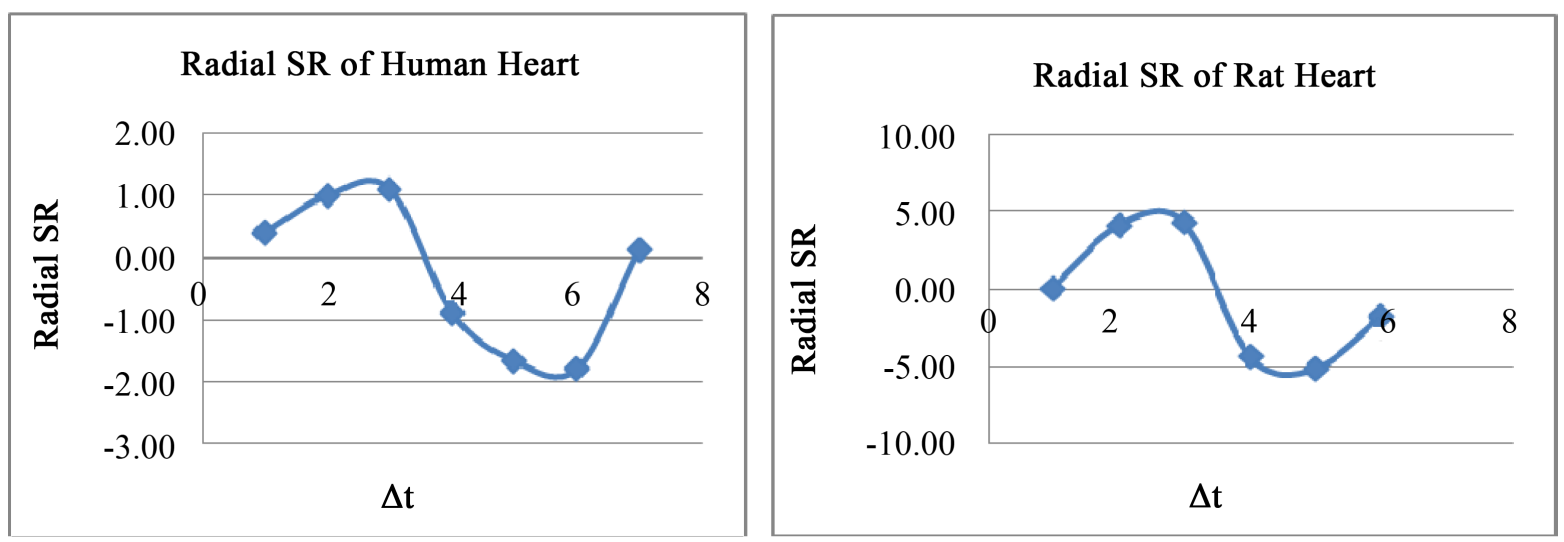

Figure 8. The radial SR change of human and rat heart during cardiac cycle.

\section{Conclusions}

This study demonstrated the accuracy of using tagged magnetic resonance imaging (tMRI) to evaluate the left ventricle motion. A measure of interest characterizing certain aspect of the LV wall biomechanics, such as strain, SR can be derived and expressed analytically. Any deviation in such measure from its normative value would indicate cardiac dysfunction, and thereby served as biomarker for detecting cardiac abnormalities and evaluating its severity in clinical investigations.

Active research focuses on determining specific and sensitive biomarkers at global or regional level for quantitatively evaluating the LV performance under normal or pathological conditions. Further studies is needed to evaluate other indicator of the heart function such as rotation and torsion of left ventricle using tMRI technique to approve the ability and capability of this noninvasive technique in evaluating the cardiac function for different species.

One important application is in evaluating diabetic heart where the myocardial tissue contraction is affected due to the fibrotic tissue deposition. Other applications are cases where the heart goes through remodeling after infarct or myocardial hypertrophy.

\section{Study Limitation}

The current study was performed on a limited number of subjects. By increasing the number of subjects and performing statistical analysis on the Radial Strain and radial SR can yield group averages to better embody the mean biomechanics of the $\mathrm{LV}$ wall motion as normative measures in healthy individuals.

Tag fading in human tMRI cardiac images decreases the image quality during the cardiac cycle which makes the radial strain and strain rate calculation more difficult; another limitation is the number of subject (9 subjects) which is due to the difficulties of acquiring the tMRI data and the long time needed for this technique. Future study is needed on diseased heart using this technique to compare control and diseased heart radial strain and strain rate in order to validate this method on dysfunction heart.

\section{Acknowledgements}

This work was supported by a grant number 231/34 from Aljouf University.

\section{References}

[1] Bogaert, J. and Rademakers, F.E. (2001) Regional Non-Uniformity of Normal Adult Human Left Ventricle. AJP Heart and Circulatory Physiology, 280, H610-H620.

[2] Sengupta, P.P., Korinek, J., Belohlavek, M., et al. (2006) Left Ventricular Structure and Function: Basic Science for Cardiac Imaging. Journal of the American College of Cardiology, 48, 1988-2001. http://dx.doi.org/10.1016/j.jacc.2006.08.030

[3] Rüssel, I.K., Götte, M.J.W., Bronzwaer, J.G., Knaapen, P., Paulus, W.J. and van Rossum, A.C. (2009) Left Ventricular Torsion an Expanding Role in the Analysis of Myocardial Dysfunction. JACC: Cardiovascular Imaging, 2, $648-655$ 
[4] Delgado, V., Ypenburg, C., van Bommel, R.J., Tops, L.F., et al. (2008) Assessment of Left Ventricular Dyssynchrony by Speckle Tracking Strain Imaging Comparison Between Longitudinal, Circumferential, and Radial Strain in Cardiac Resynchronization Therapy. Journal of the American College of Cardiology, 51, No. 20

[5] Becker, M., Kramann, R., Franke, A., et al. (2007) Impact of Left Ventricular Lead Position in Cardiac Resynchronization Therapy on Left Ventricular Remodelling. A Circumferential Strain Analysis Based on 2D Echocardiography. European Heart Journal 28, 1211-1220. http://dx.doi.org/10.1093/eurheartj/ehm034

[6] Kouzu, H., Yuda, S., Muranaka, A., et al. (2010) Left Ventricular Hypertrophy Causes Different Changes in Longitudinal, Radial, and Circumferential Mechanics in Patients with Hypertension: A Two-Dimensional Speckle Tracking Study. Journal of the American Society of Echocardiography, 24, No. 2.

[7] Shi, J., Alenezy, M., Smirnova, I.V. and Bilgen, M. (2012) Construction of a Two-Parameter Empirical Model of Left Ventricle Wall Motion Using Cardiac Tagged Magnetic Resonance Imaging Data. BioMedical Engineering OnLine, 11, 79.

[8] D’hooge, J., Heimdal, A., Jamal, F., Kukulski, T., Bijnens, B., Rademakers, F., Hatle, L., Suetens, P. and Sutherland, G.R. (2000) REVIEW ARTICLE Regional Strain and SR Measurements by Cardiac Ultrasound: Principles, Implementation and Limitations. European Journal of Echocardiography, 1, 154-170. http://dx.doi.org/10.1053/euje.2000.0031

[9] Loganathan, R., Bilgen, M., Al-Hafez, B., Alenezy, M.D. and Smirnova, I.V. (2006) Cardiac Dysfunction in the Diabetic Rat: Quantitative Evaluation Using High Resolution Magnetic Resonance Imaging. Cardiovascular Diabetology, 5, 7. http://dx.doi.org/10.1186/1475-2840-5-7

[10] http://rsb.info.nih.gov/ij/download.html

[11] Moore, C.C., Lugo-Olivieri, C.H., McVeigh, E.R. and Zerhouni, E.A. (2000) Three-Dimensional Systolic Strain Patterns in the Normal Human Left Ventricle: Characterization with Tagged MR Imaging Radiology. Radiology, 214, 453-466.

[12] Holinski, S., Knebel, F., Heinze, G., Konertz, W., Baumann, G. and Borges, A.C. (2011) Disclosures Noninvasive Monitoring of Cardiac Function in a Chronic Ischemic Heart Failure Model in the Rat Assessment With Tissue Doppler and Non-Doppler 2D Strain Echocardiography. Cardiovascular Ultrasound, 9, 9, 15.

[13] Yuan, L.-J., Takenaka, K., et al. (2014) Normal and Shear Strains of the Left Ventricle in Healthy Human Subjects Measured by Two-Dimensional Speckle Tracking Chocardiography. Cardiovascular Ultrasound, $12,7$. http://dx.doi.org/10.1186/1476-7120-12-7

[14] Koshizuka, R., Ishizu, T., Kameda, Y., Kawamura, R., Seo, Y. and Aonuma, K. (2013) Longitudinal Strain Impairment as a Marker of the Progression of Heart Failure with Preserved Ejection Fraction in a Rat Model. Journal of the American Society of Echocardiography, 26, 316-323. http://dx.doi.org/10.1016/j.echo.2012.11.015

[15] Kuijer, J.P., Marcus, J.T., Götte, M.J., van Rossum, A.C. and Heethaar, R.M. (2002) Three-Dimensional Myocardial Strains at End-Systole and during Diastole in the Left Ventricle of Normal Humans. Journal of Cardiovascular Magnetic Resonance, 4, 341-351. http://dx.doi.org/10.1081/JCMR-120013299

[16] Weytjens, C., Cosyns, B., D’hooge, J., Gallez, C., Droogmans, S., Lahoute, T., Franken, P. and Camp, G.V. (2006) Doppler Myocardial Imaging in Adult Male Rats: Reference Values and Reproducibility of Velocity and Deformation Parameters. 